\title{
Parametric RBAC Maintenance via Max-SAT
}

\author{
Marco Benedetti \\ Bank of Italy, ICT Department \\ Centro Donato Menichella, Roma \\ marco.benedetti@bancaditalia.it
}

\author{
Marco Mori \\ Bank of Italy, ICT Department \\ Centro Donato Menichella, Roma \\ marco.mori@bancaditalia.it
}

\begin{abstract}
In the past decade, many organizations have adopted a Role-Based Access Control model (RBAC) to reduce their administration costs and increase security. The migration to $\mathrm{RBAC}$ requires a role engineering phase aimed at generating "good" initial roles starting from direct assignments of permissions to users. For an RBAC approach to be effective, however, it is also necessary to update roles and keep them compliant with the dynamic nature of the business processes; not only this, but errors and misalignments between the current RBAC state and reality need to be promptly detected and fixed.

In this paper, we propose a new maintenance process to fix and refine an RBAC state when "exceptions" are detected. Exceptions are permissions some users realize they miss that are instrumental to their job and should be granted as soon as possible. They are catched by a monitoring system as unexpected "access denied" conditions and then validated by the RBAC administrator. The fix we produce aims at balancing two conflicting objectives, i.e., (i) simplifying the current RBAC state, and (ii) reducing the transition cost. Our approach is based on a Max-SAT formalization of this trade-off and it exploits incomplete solvers that quickly provide approximations of optimal solutions.
\end{abstract}

Experiments show good performance on real-world benchmarks.

Disclaimer: The opinions expressed and conclusions drawn are those of the authors and do not necessarily reflect the views of the authors' company.

\section{CCS CONCEPTS}

- Security and privacy $\rightarrow$ Logic and verification; Access control; Authorization;

\section{KEYWORDS}

Role Maintenance; Role Based Access Control (RBAC); Max-SAT

\section{ACM Reference Format:}

Marco Benedetti and Marco Mori. 2018. Parametric RBAC Maintenance via Max-SAT. In SACMAT '18: The 23rd ACM Symposium on Access Control Models \& Technologies (SACMAT), June 13-15, 2018, Indianapolis, IN, USA. ACM, New York, NY, USA, 11 pages. https://doi.org/10.1145/3205977.3205987

Permission to make digital or hard copies of all or part of this work for personal or classroom use is granted without fee provided that copies are not made or distributed for profit or commercial advantage and that copies bear this notice and the full citation on the first page. Copyrights for components of this work owned by others than ACM must be honored. Abstracting with credit is permitted. To copy otherwise, or republish, to post on servers or to redistribute to lists, requires prior specific permission and/or a fee. Request permissions from permissions@acm.org.

SACMAT '18, fune 13-15, 2018, Indianapolis, IN, USA

(C) 2018 Association for Computing Machinery.

ACM ISBN 978-1-4503-5666-4/18/06 ..\$15.00

https://doi.org/10.1145/3205977.3205987

\section{INTRODUCTION}

Complex organizations have to guarantee the correct access to software, systems, and data to a large number of employees involved in heterogeneous and evolving tasks. Classical access control policies based on a direct assignment of permissions to users have shown their limits: Granting and revoking individual permissions as tasks evolve and employees move is inefficient and error-prone.

Role Based Access Control - RBAC [3] has emerged as a solution to this issue and is currently widely adopted; it simplifies the management of permissions by defining roles, which include the permissions required to execute certain tasks. Users gain the permissions which are included in at least one of the roles assigned to them. In case users are moved to different tasks or if tasks change, it is sufficient to operate at the role level without modifying several Permission-to-User assignments. In addition, basic security principles such as Least Privilege and Separation of Duties may be easily enforced by posing conditions on roles and their assignments.

In order for an organization to adopt the RBAC model, it has to implement a role management lifecycle $[14,15]$ process, aimed at designing, implementing, and maintaining roles. To design (initial) roles, it is necessary to analyze Permission-to-User assignments and group together permissions into functional roles compactly representing the current access control policy. This is typically done via an automatic heuristic process called role mining [8]. While role mining supports the discovery of roles from scratch, in many cases organizations need to just tune or maintain the current set of roles: Roles may have to be revised to support new User-to-Permission assignments; or, a (possibly hand-made) RBAC state may contain errors, which need to be first discovered and then incorporated into the current state (after validation by a security administrator).

While tuning an RBAC model, the company has to consider conflicting objectives. It certainly benefits from a simplification of the current Permission-to-Role and Role-to-User assignments, i.e., of the so called RBAC state. Different metrics have been proposed in the literature to characterize the complexity of an RBAC state; they are often based on a combination of the number of roles and the number of assignments in it $[1,9]$. Nevertheless, any uninformed simplification of a given RBAC state may heavily modify the current assignments, thus disrupting organizational processes and/or separation of duty constraints [13], not to mention the muscle memory of any human actor involved in managing and assigning roles. This may be acceptable if the organization is very small or at a preliminary stage of RBAC adoption; not so much in case of a large company with its own well-defined, perhaps "cherished" working set of roles, which have been costly defined and negotiated.

In this paper we define a new parametric RBAC maintenance process that supports both from-scratch design and maintenance of an existing role model. When performing maintenance, the in-place RBAC state is provided as input to the algorithm (together with 
new requirements to satisfy) and an adjusted model is produced as output, which accommodates the new requirements by "patching" the original state. When starting with a clean slate, a fully compliant RBAC state is grown out of the empty state by a sequence of patches.

As a key feature, the new algorithm aims at balancing two possibly conflicting metrics: similarity and simplicity. Similarity is a measure of how different the patched state is from the pre-patch state: the closer the two states the less disrupting the update. Simplicity is a measure of how close the patched state is to the unconstrained, optimal RBAC state: the more a patch strives for optimality, the larger the potential impact on the organization. As we will see, in our approach the RBAC administrator is provided with means to strike the best balance between these two objectives.

The algorithm generates Max-SAT [6] instances and solves them via publicly-available, state-of-the-art solvers. Several well known datasets are adapted to this new incremental setting and are used to establish the practicality of our solution in real-world cases.

The rest of this paper is organized as follows. Section 2 frames the problem and presents a working example. Section 3 defines the Max-SAT formalization and introduces two metrics we use to evaluate the quality of an RBAC state. Section 4 describes the datasets we use in the experiments and presents the results of our experimental evaluation. Section 5 discusses related works. Section 6 concludes the paper and proposes directions for future work.

\section{PARAMETRIC RBAC MAINTENANCE}

\subsection{Preliminaries}

Role mining is a preliminary step to enable the adoption of an RBAC model. It can be executed bottom-up or top-down.

The bottom-up approach takes as input an existing set of direct Permission-to-User assignments. Then, mining roles consists in defining a collection of roles (sets of permissions) and then assigning one or more roles to each user. The permissions each user is granted by any of his role(s) have to be exactly the same Permissions-to-User assignments he had before roles were introduced.

Formally, starting from a binary matrix $U P A \in\{0,1\}^{m, n}$ representing the assignments of $n$ permissions to $m$ users, role mining factorizes it into a Role-to-User matrix $U A \in\{0,1\}^{m, k}$ and a Permission-to-User matrix $P A \in\{0,1\}^{k, n}$, for some positive integer $k$ (number of roles), such that $U A \otimes P A=U P A$, where the boolean matrix multiplication operator $\otimes$ is defined as $U P A_{i j}=$ $\bigvee_{l=1}^{k}\left(U A_{i l} \wedge P A_{l j}\right)$.

Top-down approaches-employed as an alternative or in combination with bottom-up mining-define roles starting from an analysis of the business processes to support. Top-down roles are typically hand-crafted by business stakeholders after an analysis of the organization processes and of the permissions users require to execute them. Hand-made roles, though validated and closely inspected, may of course contain (several) errors.

Whether the synthesis is top-down or bottom-up, once an $R B A C$ state is in place, a role maintenance process is required to adapt the state to intervening organizational changes (employees move, new systems are deployed, etc.) or to fix errors that become apparent during actual usage.

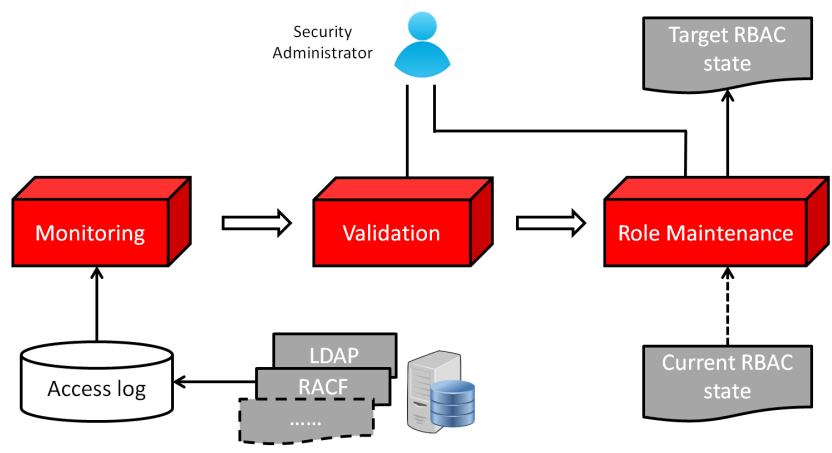

Figure 1: The Parametric Role Maintenance process.

\subsection{Maintenance of an RBAC state}

Errors show up as either Permission-to-User assignments that are not implied by the current state but should be, or as unnecessary permissions granted to users. In this paper, we address missing permissions only, though the formalization in Section 3 is capable of dealing with extra permissions (the issue with extra permissions is how to discover them in the first place, a subject out of scope here). Figure 1 describes the process of discovering missing permissions. The logs captured by the monitoring systems record (among the other things) events corresponding to missing permissions. The security administrator comes into play at this stage by validating prohibited accesses; of particular interest are false positives, i.e., permissions a user misses but should be allowed to have, which we call exceptions. The administrator may also directly specify further exceptions by generalizing the issue at hand.

Starting from a (validated) exception $e: p \rightarrow u$ to be incorporated into an existing RBAC state $S_{0}$, the maintenance phase generates a target RBAC state with the following properties:

(a) it is semantically equivalent to $S_{0}$, aside from accommodating exception $e$, i.e., $u$ now has permission $p$;

(b) among all states that satisfy (a), it is either (i) the most similar to $S_{0}$, or (ii) the simplest possible state, or (iii) any state in between these two extremes.

Section 3 formalizes the meaning of "most similar", "simplest possible", and "in between". Maintenance is applied as new exceptions arrive. If necessary, several exceptions may be dealt with at once, in a single step (however, we only show the exception-by-exception workflow in the following sections). The degree of freedom at (b) is exploited by the security administrator to strike the best balance between evolving the state towards simplicity and preserving the status quo, according to criteria external to the algorithm.

\subsection{Scope of application}

Role maintenance may also act as a fixing or mining tool, depending on how it is applied; indeed, the state fed into it can be:

Fix A hand-made, top-down RBAC state, which despite possibly containing (several) errors cannot be radically overhauled because it has been agreed on by several stakeholders; here, role maintenance is basically used to gradually shepherd the hand-made model to the reality of business processes; 
Tune An in-place, fully satisfactory RBAC state which needs to undergo maintenance to accommodate exogenous events, such as updates in business processes, deployment/replacement of information systems, relocation of employees, etc.;

Mine An empty state granting no permission to anyone; in this case, role maintenance is used as an incremental role mining procedure whereby the administrator generates a wellbehaved and well-structured RBAC state by monitoring missing permissions on-line and judiciously granting them.

We focus on the "tune" use case in the rest of the paper.

\subsection{A working example}

Let us consider a consulting firm with dozens of employees. They use two applications that store data into a company database. The first application supports the publishing activity of the company. The second application is used for marketing purposes, specifically in the definition of campaigns for mainstream media. An independent in-house quality assurance process ensures the compliance of the actions taken through the publishing and the marketing platform with all relevant guidelines and regulations. Employees also use a general-purpose Internet service and an in-house e-mail server, through which they communicate with institutional stakeholders. An Intranet is available for internal communication and to access business applications. The HR department manages a database with employees' data. A web site describes the services offered by the company on the web. The application server and the databases are managed by different administrators.

An RBAC solution is in place at this company: Roles have been crafted so that each employee is enabled to perform only the task(s) he is involved in. The set of (business and technical) roles are: publishing, marketing, quality assurance, general communication, business communication, HR management, server administration and database administration. As usual, each role is associated with the set of permissions required to carry out the corresponding task(s). For example, the marketing (publishing) role entails the permission to access the marketing (publishing) application, in addition to the permission to access the shared business database. The business communication role grants the permission to use the mail server and the Intranet, while the general communication role gives access to the Internet. Finally, the database administration role is required to operate on the business and the HR databases.

Let us suppose the RBAC manager at this company is now faced with these urgent requests: An employee is about to join the marketing division and needs the corresponding permissions; at the same time, another employee who is already in that division just realized he lacks some much needed permissions to operate; another employee from the marketing division is required to (also) work in the publishing process for a while. Finally, yet another employee is to be temporarily granted permissions to help the database administrator, but only on a non-critical dataset.

The RBAC administrator would like to be supported in the process of re-configuring the RBAC state. In particular, he would like to automatically obtain good answers to questions such as:

- "How to modify the current RBAC state to implement the necessary variations and nothing more?"
- "Is it possible to operate on the Permission-to-Role and/or Roleto-User assignments without adding new roles?"

- "Would it be better to just create new custom roles?"

- "Is this an occasion to merge some existing roles into less and more clear-cut business figures?"

- "Once I'm at it, can I simplify the current role set while keeping the solution stable w.r.t. to the desired RBAC state?"

\section{FORMALIZATION}

We use the language of propositional logic to represent RBAC maintenance instances. The question we ask is not simply about the satisfiability of a logic statement by some propositional model (SAT), because we need to also express preferences between conflicting objectives (similarity and simplicity of the output RBAC state). It turns out a slightly different, "optimizing" framework perfectly suited to our needs is Max-SAT, which considers all possible truth assignments to the input formula and picks the best one according to a fitness metric defined in the Max-SAT language itself.

\subsection{SAT and Max-SAT}

A SAT problem [2] is solved by assigning a truth value in $\{$ True, False $\}$ to each Boolean variable that appears in the input propositional formula in such a way that the formula as a whole evaluates to True. If at least one such assignment exists, the formula is satisfiable and the satisfying assignment is called a model; otherwise, the formula is unsatisfiable (inconsistent) and it has no model. This problem is intractable (NP-complete) in general, yet several highly efficient solvers exist that in practice solve real-world problems with millions of variables in reasonable time.

Given any $U P A$ matrix, its factorization into $U A \otimes P A=U P A$ for some number of roles $k$ can be directly expressed in SAT as:

$$
\bigwedge_{i j}\left[U P A_{i j} \leftrightarrow \vee_{l=1}^{k}\left(U A_{i l} \wedge P A_{l j}\right)\right]
$$

(1) is a SAT instance with $k(m+n)$ variables. Without loss of generality, most SAT solvers require input formulas in Conjunctive Normal Form (CNF), i.e., a conjunction of clauses, each clause being a disjunction of literals. By either reworking the formula (possibly enlarging its size) or adding auxiliary variables, it is possible to rewrite any non-CNF problem like (1) in CNF.

In SAT, all clauses have to be satisfied for the formula to be declared satisfiable. The Maximum Satisfiability problem (Max-SAT) is a variant of SAT that relaxes this premise. The goal of MaxSAT is to find an assignment that makes true the largest possible subset of clauses: some may remain unsatisfied. Partial Max-SAT is another variant in which some clauses are declared hard and must be satisfied no matter what, as in SAT, while others are declared soft: As many of them as possible must be satisfied, à la Max-SAT. Yet another variant is Weighted Max-SAT, that generalizes MaxSAT by associating a positive real or integer weight to each input clause: An assignment that maximizes the sum of the weights of satisfied clauses, rather than just their number, is sought (clauses left unsatisfied contribute nothing to the sum).

Finally, the Weighted Partial Max-SAT (WPMS) language combines both features: It finds an assignment that satisfies all hard clauses while maximizing the cumulated weight of satisfied soft clauses. We employ WPMS here (and call it simply Max-SAT). 


\subsection{Hard constraints}

Hard clauses are well suited to represent invariants that must hold on any RBAC state we may possibly output, and in particular to capture condition (a) from Section 2.2.

The input RBAC state is represented by two boolean matrices $U A^{0}$ and $P A^{0}$ such that $U A^{0} \otimes P A^{0}=U P A^{0} . U A^{0}$ and $P A^{0}$ are respectively a $m \times k^{0}$ and a $k^{0} \times n$ boolean matrices having non-empty roles, i.e., $\forall_{t=1, \ldots, k^{0}}\left(\bigvee_{i=1, . ., m} U A_{i, t}=\right.$ True $) \wedge\left(\bigvee_{j=1, \ldots, n} P A_{t, j}=\right.$ True $)$ Given an exception $e: p_{i} \rightarrow u_{j}$ meant to grant a new permission $p_{i}$ to user $u_{j}$ (it was $U P A_{i, j}^{0}=$ False), we define EXC(e) as the $m \times n$ boolean matrix that is True at $(i, j)$ and False everywhere else. We then need $k(m+n)$ variables to represent the unknown elements of the updated Role-to-User $(U A)$ and of the Permissions-to-Role $(P A)$ matrices, where $m, k$, and $n$ are the number of users, roles, and permissions.

We have to find two boolean matrices $U A$ and $P A$ such that:

$$
U A \otimes P A=U P A^{0} \oplus E X C(e)=U P A
$$

where $\oplus$ is the boolean matrix sum operator. In terms of the variables defining UA and PA, this can be written:

$\bigwedge_{i, j} F_{i, j}$, where $F_{i, j}:=\left(\vee_{t \in[1, k]} U A_{i, t} \wedge P A_{t, j}\right)=U P A_{i, j}^{0} \vee E X C_{i, j}(e)$

Formulas $F_{i, j}$ are not in CNF. To turn them into CNF we observe that only two cases have to be considered:

$$
\begin{gathered}
\left(\begin{array}{ccc}
\ldots & \ldots & \ldots \\
U A_{i_{0}, 1} & \ldots & U A_{i_{0}, k} \\
\vdots & \vdots & \vdots \\
U A_{i_{1}, 1} & \ldots & U A_{i_{1}, k} \\
\ldots & \ldots & \ldots
\end{array}\right)\left(\begin{array}{ccccc}
\ldots & P A_{1, j_{0}} & \ldots & P A_{1, j_{1}} & \ldots \\
\vdots & \vdots & \vdots & \vdots & \vdots \\
\ldots & P A_{k, j_{0}} & \ldots & P A_{k, j_{1}} & \ldots
\end{array}\right)= \\
i_{0}\left(\begin{array}{ccccc}
\ldots & \ldots & \ldots & \ldots & \ldots \\
\ldots & \text { False } & \ldots & \ldots & \ldots \\
\vdots & \vdots & \vdots & \vdots & \vdots \\
\ldots & \ldots & \ldots & \text { True } & \ldots \\
\ldots & \ldots & \ldots & \ldots & \ldots
\end{array}\right)
\end{gathered}
$$

If $U P A_{i, j}^{0} \vee E X C_{i, j}$ is False, such as at index $\left(i_{0}, j_{0}\right)$ above, we have:

$$
\left[\bigvee_{t \in[1, k]}\left(U A_{i, t} \wedge P A_{t, j}\right)\right]=\text { False }
$$

which can be rewritten as a set of clauses by negating both sides:

$$
\operatorname{has}_{i, j}(U A, P A):=\bigwedge_{t \in[1, k]}\left(\neg U A_{i, t} \vee \neg P A_{t, j}\right)
$$

If $U P A_{i, j}^{0} \vee E X C_{i, j}$ is True, such as at index $\left(i_{1}, j_{1}\right)$, we have:

$$
\left[\bigvee_{t \in[1, k]}\left(U A_{i, t} \wedge P A_{t, j}\right)\right]=\text { True }
$$

Here we apply the Tseytin transformation [12] by inserting $k$ auxiliary variables $\left\{a u x_{1}, \ldots, a u x_{k}\right\}$, one for each disjunct:

$$
\left\{\begin{array}{l}
\bigvee_{t \in[1, k]} \text { aux }_{t} \\
\bigwedge_{t \in[1, k]} \text { aux }_{t} \leftrightarrow\left(U A_{i, t} \wedge P A_{t, j}\right)
\end{array}\right.
$$

which is equivalent to the CNF form:

hasnt $_{i, j}(U A, P A):=\left\{\begin{array}{l}\vee_{t \in[1, k]} \text { aux }_{t} \\ \bigwedge_{t \in[1, k]}\left(\neg \operatorname{aux}_{t} \vee U A_{i, t}\right) \wedge\left(\neg a u x_{t} \vee P A_{t, j}\right) \\ \bigwedge_{t \in[1, k]}\left(\text { aux }_{t} \vee \neg U A_{i, t} \vee \neg P A_{t, j}\right)\end{array}\right.$

Overall,the hard clauses that guarantee we are incorporating the exception(s) in EXC are:

$$
\operatorname{exc}(U A, P A):=\bigwedge_{i, j \mid U P A_{i, j}} \operatorname{has}_{i, j}(U A, P A) \quad \wedge \bigwedge_{i, j \mid \neg U P A_{i, j}} \operatorname{hasnt}_{i, j}(U A, P A)
$$

\subsection{Soft constraints}

We use soft clauses to express contraints that we know are in tradeoff and cannot always be satisfied simultaneously. In particular, we want to capture the conditions (i) and (ii) at point (b) in Section 2.2.

Identity. To express the condition that the output RBAC state $(U A, P A)$ must be identical to the input state $\left(U A^{0}, P A^{0}\right)$ we write:

$$
\left\{\begin{array}{c}
\bigwedge_{i \in[1, m], j \in[1, k]} U A_{i, j} \leftrightarrow U A_{i, j}^{0} \\
\bigwedge_{i \in[1, k], j \in[1, n]} P A_{i, j} \leftrightarrow P A_{i, j}^{0}
\end{array}\right.
$$

which in $\mathrm{CNF}$ is:

$$
e q(U A, P A):=\left\{\begin{array}{l}
\bigwedge_{i, j}\left(U A_{i, j} \vee \neg U A_{i, j}^{0}\right) \wedge\left(\neg U A_{i, j} \vee U A_{i, j}^{0}\right) \\
\bigwedge_{i, j}\left(P A_{i, j} \vee \neg P A_{i, j}^{0}\right) \wedge\left(\neg P A_{i, j} \vee P A_{i, j}^{0}\right)
\end{array}\right.
$$

Note that $\operatorname{exc}(U A, P A) \wedge e q(U A, P A)$ is unsatisfiable by construction in the traditional SAT sense.

Sparsification. To express the condition that the output RBAC state $(U A, P A)$ must be as "simple" as possible we need to adopt some notion of simplicity. The two simplifying conditions we consider are: (i) a reduction in the number of Roles-to-User and/or Permission-to-Role assignments ("sparsification"), and (ii) a reduction in the number of roles (see next section, "Contraction").

According to (i), the sparsest possible RBAC state would be:

$$
\operatorname{sparse}(U A, P A):=\left\{\begin{array}{l}
\bigwedge_{i \in[1, m], j \in[1, k], U A_{i, j}^{0}=1} \neg U A_{i, j} \\
\bigwedge_{i \in[1, k], j \in[1, n], P A_{i, j}^{0}=1} \neg P A_{i, j}
\end{array}\right.
$$

Contraction. Sometimes, incorporating an exception makes it possible to satisfy equation (2) with one less role than those present in $\left(U A^{0}, P A^{0}\right)$. The soft constraint sparse $(U A, P A)$ is not sufficient to prefer a reduction in the number of roles over similarly-sized but sparse unassignments from $U A$. The fact that role $j$ is no longer in use in the target RBAC state is represented by a column of 0 's in the Role-to-User assignment matrix $(U A)$; so the condition is:

$$
\operatorname{contr}(U A):=\bigwedge_{j \in[1, k]} \text { unused }_{j}(U A)
$$

where $\left\{\right.$ unused $\left._{j}\right\}$ are auxiliary variables telling whether role $j$ is assigned to someone (unused $_{j}=$ False $)$ or not $\left(\right.$ unused $_{j}=$ True ):

$$
\text { unused }_{\text {contr }}(U A):=\bigwedge_{j \in[1, k]}\left[\text { unused }_{j}(U A) \leftrightarrow \wedge_{t \in[1, m]} \neg U A_{t, j}\right]
$$

Expansion. Dually, it is possible that the only way to incorporate an exception is by adding one role to the state, i.e., one column 
to $U A$ and one row to $P A$. In a propositional encoding, the only way to accommodate for a possible expansion of the matrices we handle is to "reserve space" in advance for the additional row and column. This means we actually work with an RBAC state made by a matrix $U A^{+}$with $k+1$ columns and a matrix $P A^{+}$with $k+1$ rows. The condition that this additional role stays unused is:

$$
\text { noexp }(U A):=\text { unused }_{k+1}
$$

where unused $_{k+1}$ is an auxiliary variable telling whether the additional role remains unused:

$$
\begin{aligned}
& \text { unused }_{\exp }(U A):=\text { unused }_{k+1} \leftrightarrow \wedge_{i \in[1, m]} \neg U A_{i, k+1}^{+}
\end{aligned}
$$

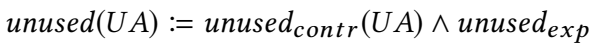

In an encoding where both contraction and expansion constraints are added, we enforce a coherent interplay between the two opposite effects by saying they must not happen simultaneously:

$$
\operatorname{mutex}(U A):=\operatorname{noexp}(U A) \vee \neg \text { leastcontr }(U A)
$$

where leastcontr tells weather at least a role is no longer in use:

$$
\text { leastcontr }(U A):=\bigvee_{j \in[1, k]} \operatorname{unused}_{j}(U A)
$$

\subsection{The complete encoding}

We have all the pieces to present the complete encoding of the RBAC maintenance problem as a Weighted Partial Max-SAT instance. First, let us list the hard constraints (HC) that any feasible solution must comply with. In addition to those from Section 3.2, all the auxiliary contraints from Section 3.3 are hard, because auxiliary propositional variables cannot cause inconsistencies on their own, yet they must always be assigned a consistent value:

$$
H C(U A, P A):=\operatorname{exc}\left(U A^{+}, P A^{+}\right) \wedge \operatorname{unused}(U A) \wedge \operatorname{mutex}(U A)
$$

Soft constraints (SC) are used to express and balance two potentially conflicting objectives of the RBAC maintenance procedure:

(1) maximizing the similarity between the target and the origin RBAC state; in this sense, the higher the weight we assign to clauses in (6), the better;

(2) maximizing the simplicity of the target RBAC state (independently of the origin RBAC state); in this sense, the higher the weight we assign to clauses in (7), (8), and (10), the better.

Let us assume the weight of a given clause $C$ is a real value ${ }^{1}$ $w \in[0,1]$, noted $w: C$. The notation $w:\left(C_{1} \wedge \ldots \wedge C_{n}\right)$ is a shorthand for $w / n: C_{1} \wedge \ldots \wedge w / n: C_{n}$, while $w_{1}:(w 2: C)$ is interpreted as $\left(w_{1} \cdot w_{2}\right): C$. The three components (7), (8), and (10) of the "simplicity" objective are merged into one weighted set of clauses, noted $S I M P_{k^{+}, k^{-}}(U P, P A)$ and defined as:

$$
v:\left[k^{-}: \operatorname{contr}(U A, P A) \wedge \operatorname{sparse}(U A, P A) \wedge k^{+}: \operatorname{noexp}(U A, P A)\right]
$$

Here, $k^{-} \geq 0$ is a parameter meant to quantify how much we reward the elimination of a role versus to the sparsification of the matrix; $k^{+} \geq 0$ measures our adversion to introduce a new role ${ }^{1}$ Some Max-SAT solvers only accept positive integer values as weights; we can map
real values onto integers that are equivalent to the effect of the optimization we
perform as weight $t_{\text {int }}=\left\lceil\delta *\right.$ weight $\left._{\text {real }}\right\rceil$ where $\delta$ is the minimum absolute
difference between the sum of the weights of any two disjoint subsets of the real weights mentioned in the formula. if not strictly necessary. $v:=\left(1+k^{-}+k^{+}\right)^{-1}$ is a normalization factor meant to ensure the weights of the three components sum up to 1 . We assume $k^{-}>k^{+}>1$, i.e., we value the possibility to expunge a role more than the removal of an equivalent number of assignments; adding a role is a last resort.

Finally, let $\beta \in[0,1]$ be a balancing parameter that measures the extent to which the RBAC administrator is interested in a simplified ( $\beta$ closer to 1 ) VS a stable ( $\beta$ closer to 0 ) RBAC state; we pose:

$S C_{\beta, k^{-}, k^{+}}(U A, P A):=(1-\beta): e q(U A, P A) \wedge \beta: S I M P_{k^{+}, k^{-}}(U A, P A)$

\subsection{Solutions and their quality}

Given (i) the input RBAC state $\left(U A^{0}, P A^{0}\right)$, (ii) the exception matrix $E X C$ to incorporate, and (iii) some specific values for the parameters $\beta, k^{+}$, and $k^{-}$, we submit to a Max-SAT solver the hard clauses (14) plus the soft clauses (16). The Max-SAT solver returns a fixed RBAC state $(U A, P A)$, i.e., a truth assignment to all the $k(m+n)$ variables that define $U A$ and $P A$. Note that the encoding is satisfiable by construction, so we always obtain a fixed state.

To assess the quality of $U A, P A$ (both per se and w.r.t. $U A^{0}, P A^{0}$ ) we need some synthetic indicators for the two dimensions we are after: simplicity and similarity.

Similarity is computed according to the metric defined in [13], which is based on the Jaccard coefficient.

Role-to-Role similarity. The similarity between two roles $r_{1}$ and $r_{2}$ granting permissions $P_{1}$ and $P_{2}$ respectively is defined as:

$$
\operatorname{sim}_{1-1}\left(r_{1}, r_{2}\right):=\frac{\left|P_{1} \cap P_{2}\right|}{\left|P_{1} \cup P_{2}\right|}
$$

Role-to-RoleSet similarity. The similarity between a role $r$ and a set of roles $R$ is defined as:

$$
\operatorname{sim}_{1-N}(r, R):=\max _{r_{x} \in R} \operatorname{sim}_{1-1}\left(r, r_{x}\right)
$$

RoleSet-to-RoleSet similarity. The similarity between a set of roles $R_{1}$ and a set of roles $R_{2}$ is defined as:

$$
\operatorname{sim}_{N-N}\left(R_{1}, R_{2}\right):=\operatorname{avg}_{r \in R_{1}} \operatorname{sim}_{1-N}\left(r, R_{2}\right)
$$

Building on these definitions, we pose:

Similarity. The similarity of the target RBAC state (with roles $R$ ) to the source RBAC state (with roles $R_{0}$ ) as:

$$
\operatorname{sim}\left(R_{0}, R\right):=\frac{\operatorname{sim}_{N-N}\left(R_{0}, R\right)+\operatorname{sim}_{N-N}\left(R, R_{0}\right)}{2}
$$

This similarity function is always between 0 and 1 ; in particular, the value 1 is obtained iff $R \equiv R_{0}$.

Simplicity is defined in different ways in the relevant literature; metrics taken into account to define it include, but are not limited to, the number of roles, assignments, erroneous permission granted, relations among roles, permission assigned directly, and any combination of these metrics. We start from a definition of "absolute complexity" that grows with the number of roles and the number of assignments in $U A$ and $P A$ :

$$
\operatorname{comp}(U A, P A):=(|U A|+|P A|)+|R|^{2}
$$


where $R$ is the set of roles, and $U A$ and $P A$ are the assignments of permissions and roles ${ }^{2}$. A relative complexity is then computed as the ratio between the complexity of the target state and the complexity of the "trivial" admissible state; in such state, each user is assigned one and only one custom role which entails exactly the set of permissions that user has in UPA. In this configuration, the $U A$ matrix becomes the identity matrix, there are as many roles as users, and all the knowledge about permissions is in $P A$. The absolute complexity of such trivial state is thus $(|U P A|+|U|)+|U|^{2}$.

The (relative) simplicity ${ }^{3}$ of a state is then:

$$
o p t=1-\frac{|U A|+|P A|+|R|^{2}}{|U P A|+|U|+|U|^{2}}
$$

\section{VALIDATION}

In this section we validate our maintenance algorithm (i) at a smallscale in Section 4.1, where we apply it to our motivating example from Section 2.4; (ii) at a larger scale in Section 4.2, where we present experimental results showing its viability in real-word cases.

\subsection{Maintenance of our working example}

Table 1 and Table 2 formalize (a small version of) the example from Section 2.4. Table 3 represents the corresponding RBAC state as Permission-to-Role and Role-to-User assignments.

We now imagine that four exceptions to such initial RBAC statelisted in Table 4-are captured by the monitoring system or directly specified by the RBAC administrator. We have to incorporate them.

$e_{1}$ : According to the first exception, it is necessary to augment the permissions of user $u 4$ by granting him access to the marketing application (permission $p 7$ ). The user already has access to the publishing application and to the business database. Different RBAC states result from different values of the balance parameter $\beta$. For example, if (i) the administrator prefers not to alter the current state too much and he submits the value $\beta=0.1$ to the algorithm, the exception is incorporated by simply assigning role marketingFunct to $u 4$. The variation over the current RBAC state is minimal $(\operatorname{sim}=1)$ and the complexity of the state is almost unchanged at $o p t=0.388$ (same number of roles and the addition of a single assignment); in case (ii) the administrator values simplicity more and sets the value $\beta=0.5$, the algorithm again answers by assigning role marketingFunct to $u 4$, but then carries out further simplifying and adjustments to the input state. Namely, the busComm role is augmented with the Internet access permission thus making it possible to reduce the assignments of role genComm to just $u 6$ and $u 7$. Simplicity raises to opt $=0.432$ at the price of lowering the similarity to $\operatorname{sim}=0.958$. Let us suppose in what follows that the RBAC administrator picks option (ii).

$e_{2}$ : It is now required that the marketing application $(p 7)$ is made accessible to user $u 5$ too. Assuming (i) a high interest in similarity

\footnotetext{
${ }^{2}$ By squaring the role number, we penalize the growth of roles more than linearly compared to the role and permission assignments, without introducing arbitrary weighting factors. Alternative definitions from the literature have been tested, with minimal impact on the results from Section 4.

${ }^{3}$ Another possibility here would be to measure the absolute complexity via (18) as a percentage of the complexity of the initial state. We prefer measure (19) because it is independent of the unknown quality of the initial state (which we'll synthesize via an approximate miner) and stays in the range $[0,1)$ with a clear meaning at the extremes.
}

( $\beta=0.1$ ), the algorithm assigns role marketingFunct to $u 5$, thus maintaining a stable state $(\operatorname{sim}=1)$ and essentially the same simplicity (opt $=0.429$ ). Alternatively, (ii) the assignment $\beta=0.5$ causes the algorithm to extend the role publishingFunct-which is already assigned to $u 5-$ with the missing permission $p 7$. This is possible since no user is granted $p 8$ but not $p 7$ anymore. Thus, the new publishingFunct role enables both business applications, while marketingFunct gives access to the marketing application only, and is assigned to $u 3$. This new RBAC state has a lower similarity at $\operatorname{sim}=0.958$, but only a slightly improved simplicity at 0.446 . Since the optimization is marginal and it comes with a damage to similarity, the administrator picks option (i) over option (ii).

$e_{3}$ : User $u 3$ needs to access the publishing application (permission $p 7)$. It turns out that in the current state all users granted $p 8$ should also have $p 7$. It follows that the distinction between marketingFunct and publishingFunct makes no longer sense. It is thus not surprising that with even moderate importance assigned to simplicity $(\beta=$ 0.3 ), the algorithm answers by joining such two roles and reducing the state complexity to opt $=0.541$, at the price of some state variation $(\operatorname{sim}=0.935)$. In particular, role publishingFunct is erased and role marketingFunct, already assigned to $u 3$, is augmented with $p 8$, thus enabling the access to both business applications.

$e_{4}$ : User $u 6$ is assigned the responsibility of managing the business DB (permission p10). A role enabling the management of all the databases (business and HR, $p 10$ and $p 9$ ) already exists: $D B A d-$ min. However, $u 6$ is to be granted access to the business database only; it may be necessary to augment the role set. By applying the role-maintenance algorithm with $\beta=0.1$, we are indeed returned a target RBAC state with a new role (we call it BussDBAdmin) assigned to $u 6$ and including permission $p 10$ only. The complexity of the RBAC state is augmented since a new role is introduced (opt $=0.452)$; there is a minimal variation from the initial state $(\operatorname{sim}=0.958)$.

\subsection{Experimental Evaluation}

To experiment at a larger scale we exercise our algorithm on the semi-synthetic datasets described in Section 4.2.1. Different MaxSAT solvers are compared in Section 4.2.2 to select the one that best suits our needs. Experimental results, performed on a 20-core Intel CPU with 138GB of memory, are presented in Section 4.2.3. Unless noted otherwise, we set $k^{+}=k^{-}=1$. All the results and datasets can be downloaded from the web site [10].

4.2.1 Synthetic datasets. In order to experiment with our maintenance procedure, we require datasets that include: $(i)$ some initial RBAC state defined in terms of a set of roles and their assignment to users, and (ii) a list of exceptions: couples of Permission-to-User assignments that are either missing or in excess in such RBAC state. To the best of our knowledge, no public dataset provides such information. Most datasets only consist of a binary matrix of Permission-to-User assignments, with no associated RBAC state. Moreover, exception histories are never included.

In order to synthesize a benchmark for the maintenance problem, we start with four existing datasets: our example from Section 4.1, named SmallComp here, plus three classical problems of increasing 


\begin{tabular}{|c|lllllllllll|}
\hline $\mathrm{U} \backslash \mathrm{P}$ & $\mathrm{p} 1$ & $\mathrm{p} 2$ & $\mathrm{p} 3$ & $\mathrm{p} 4$ & $\mathrm{p} 5$ & $\mathrm{p} 6$ & $\mathrm{p} 7$ & $\mathrm{p} 8$ & $\mathrm{p} 9$ & $\mathrm{p} 10$ & $\mathrm{p} 11$ \\
\hline $\mathrm{u} 1$ & 1 & 1 & 0 & 1 & 0 & 1 & 1 & 1 & 0 & 0 & 0 \\
$\mathrm{u} 2$ & 1 & 1 & 0 & 1 & 0 & 1 & 1 & 1 & 0 & 0 & 0 \\
$\mathrm{u} 3$ & 1 & 1 & 0 & 1 & 0 & 1 & 1 & $0[1]^{3}$ & 0 & 0 & 0 \\
u4 & 1 & 1 & 0 & 1 & 0 & 1 & $0[1]^{1}$ & 1 & 0 & 0 & 0 \\
$\mathrm{u} 5$ & 1 & 1 & 0 & 1 & 0 & 1 & $0[1]^{2}$ & 1 & 0 & 0 & 0 \\
u6 & 1 & 0 & 0 & 0 & 0 & 0 & 0 & 0 & 0 & $0[1]^{4}$ & 0 \\
u7 & 1 & 0 & 0 & 0 & 0 & 0 & 0 & 0 & 0 & 0 & 0 \\
u8 & 1 & 1 & 0 & 1 & 0 & 1 & 0 & 0 & 1 & 1 & 0 \\
u9 & 1 & 1 & 0 & 0 & 0 & 1 & 1 & 1 & 0 & 0 & 1 \\
u10 & 1 & 1 & 0 & 1 & 1 & 1 & 0 & 0 & 0 & 0 & 0 \\
u11 & 1 & 1 & 1 & 0 & 0 & 1 & 0 & 0 & 0 & 0 & 0 \\
\hline
\end{tabular}

Table 1: Permission-to-User assignment matrix (UPA).

\begin{tabular}{|c|l|}
\hline Permission & Meaning \\
\hline p1 & Internet Access \\
p2 & Mail Access \\
p3 & HR DB Access \\
p4 & Business DB Access \\
p5 & High Perf. Comp. Resource Access \\
p6 & Intranet Access \\
p7 & Marketing Application Access \\
p8 & Publishing Application Access \\
p9 & HR DB Admin \\
p10 & Business DB Admin \\
p11 & Application Server Admin \\
\hline
\end{tabular}

Table 2: Permissions and their meaning.

\begin{tabular}{|c|c|c|c|c|c|c|c|c|c|c|c|c|c|c|c|c|c|c|c|c|c|c|}
\hline $\mathrm{R} \backslash \mathrm{P}$ & $\mathrm{p} 1$ & p2 & p3 & $\mathrm{p} 4$ & p5 & p6 & p7 & $\mathrm{p} 8$ & p9 & p10 & p11 & $\mathrm{u} 1$ & $\mathrm{u} 2$ & u3 & $\mathrm{u} 4$ & u5 & u6 & $\mathrm{u} 7$ & $\mathrm{u} 8$ & u9 & $\mathrm{u} 10$ & u11 \\
\hline bussComm & $0[1]^{1}$ & 1 & 0 & 0 & 0 & 1 & 0 & 0 & 0 & 0 & 0 & 1 & 1 & 1 & 1 & 1 & 0 & 0 & 1 & 1 & 1 & 1 \\
\hline genComm & 1 & 0 & 0 & 0 & 0 & 0 & 0 & 0 & 0 & 0 & 0 & $1[0]^{1}$ & $1[0]^{1}$ & $1[0]^{1}$ & $1[0]^{1}$ & $1[0]^{1}$ & 1 & 1 & $1[0]^{1}$ & $1[0]^{1}$ & $1[0]^{1}$ & $1[0]^{1}$ \\
\hline marketingFunct & 0 & 0 & 0 & 1 & 0 & 0 & 1 & $0[1]^{3}$ & 0 & 0 & 0 & 1 & 1 & 1 & $0[1]^{1}$ & $0[1]^{2}$ & 0 & 0 & 0 & 0 & 0 & 0 \\
\hline publishingFunct & 0 & 0 & 0 & 1 & 0 & 0 & 0 & 1 & 0 & 0 & 0 & $1[0]^{3}$ & $1[0]^{3}$ & $0[0]^{3}$ & $1[0]^{3}$ & $1[0]^{3}$ & $0[0]^{3}$ & $0[0]^{3}$ & $0[0]^{3}$ & $0[0]^{3}$ & $0[0]^{3}$ & $0[0]^{3}$ \\
\hline HRManagement & 0 & 0 & 1 & 0 & 0 & 0 & 0 & 0 & 0 & 0 & 0 & 0 & 0 & 0 & 0 & 0 & 0 & 0 & 0 & 0 & 0 & 1 \\
\hline QualityAssurance & 0 & 0 & 0 & 1 & 1 & 0 & 0 & 0 & 0 & 0 & 0 & 0 & 0 & 0 & 0 & 0 & 0 & 0 & 0 & 0 & 1 & 0 \\
\hline DBAdmin & 0 & 0 & 0 & 1 & 0 & 0 & 0 & 0 & 1 & 1 & 0 & 0 & 0 & 0 & 0 & 0 & 0 & 0 & 1 & 0 & 0 & 0 \\
\hline ServerAdmin & 0 & 0 & 0 & 0 & 0 & 0 & 1 & 1 & 0 & 0 & 1 & 0 & 0 & 0 & 0 & 0 & 0 & 0 & 0 & 1 & 0 & 0 \\
\hline BussDBAdmin & {$[0]^{4}$} & {$[0]^{4}$} & {$[0]^{4}$} & {$[0]^{4}$} & {$[0]^{4}$} & {$[0]^{4}$} & {$[0]^{4}$} & {$[0]^{4}$} & {$[0]^{4}$} & {$[1]^{4}$} & {$[0]^{4}$} & {$[0]^{4}$} & {$[0]^{4}$} & {$[0]^{4}$} & {$[0]^{4}$} & {$[0]^{4}$} & {$[1]^{4}$} & {$[0]^{4}$} & {$[0]^{4}$} & {$[0]^{4}$} & {$[0]^{4}$} & {$[0]^{4}$} \\
\hline
\end{tabular}

Table 3: Permission-to-Role matrix $P A$ (left) and User-to-Role matrix $U A^{T}$ (right).

dimension used in the role-mining literature, called Domino, University, and Firewall1. All these instances are defined in terms of their User-to-Permission matrix. Starting from one of these UPA matrices, we generate (purely additive) maintenance instances as follows:

(1) Given a positive integer $k$, we randomly select $k$ user permissions in $U P A$ and remove them, thus obtaining $U P A^{0}$. A random order is assigned to such permissions to obtain the list of exceptions which we ask our algorithm to incorporate;

(2) We synthesize a complete RBAC state out of $U P A^{0}$ via one of the role mining algorithms available in the literature. Given that we aim at an arbitrary (not necessarily optimal) initial state, we adopt Fast Miner, a heuristic procedure which returns a sub-optimal set of roles ${ }^{4}$. The corresponding $U A^{0}$ matrix is then iteratively generated, and the resulting RBAC state is used as initial state for the maintenance algorithm.

The resulting benchmark is described in Table 5 .

\footnotetext{
${ }^{4}$ We choose Fast Miner over the alternatives-which we tried-because it quickly returns solutions of good quality to medium and large problems. In preliminary experiments done with other miners, we observed different initial absolute values but quite similar trends (as a function of beta and of the timeout) in all the experiments.
}

RBAC state

\begin{tabular}{lcccccc} 
Exception & $\beta$ & $|U P A|$ & $|R|$ & $|U A|+|P A|$ & opt & sim \\
\hline- & - & 50 & 8 & 47 & 0.390 & - \\
$e_{1}: p 7 \rightarrow u 4$ & 0.5 & 51 & 8 & 40 & 0.432 & 0.958 \\
$e_{2}: p 7 \rightarrow u 5$ & 0.1 & 52 & 8 & 41 & 0.429 & 1 \\
$e_{3}: p 8 \rightarrow u 3$ & 0.3 & 53 & 7 & 36 & 0.541 & 0.935 \\
$e_{4}: p 10 \rightarrow u 6$ & 0.1 & 54 & 8 & 38 & 0.452 & 0.958
\end{tabular}

Table 4: List of exceptions to incorporate in the working example. In Table 1 and Table 3, we note $\operatorname{old}[n e w]^{i}$ an assignment that is changed from value old to value new by applying exception $e^{i}$.
4.2.2 Choosing a Max-SAT solver. Max-SAT solvers are either complete or incomplete. Complete solvers always identify the optimal solution (if one exists), given enough time. Incomplete solvers determine an approximate solution, with no guarantee on how distant it is from the optimum. In practice, on satisfiable non-random instances, they often produce good approximations, and quickly. Furthermore, some solvers work as anytime algorithms, i.e., given any timeout as input, they return the best solution they could possibly find (if any) within the assigned timeout.

Complete solvers. Solving large instances of the role-maintenance problem may be unfeasible in practice for complete solvers. To check if this is the case, we chose a few of the best performers at the Max-SAT 2016 international competition ${ }^{5}$. Then, we exercise these promising solvers on the datasets from Section 4.2.1, imposing a time limit of 1 hour. Results are in the following table.

\begin{tabular}{|lcccc|}
\hline Solver & SmallComp & Domino & University & Firewall1 \\
\hline Maximo & $\beta \leq 0.5$ & $\beta=0$ & $\beta=0$ & $\beta=0$ \\
MaxHS & $\beta \leq 0.4$ & $\beta=0$ & $\beta=0$ & - \\
LMHS & $\beta \leq 0.3$ & $\beta=0$ & $\beta=0$ & - \\
Ahmaxsat & $\beta \leq 0.25$ & - & - & - \\
\hline
\end{tabular}

The table shows the maximum value of $\beta$ for which solvers are able to incorporate all the exceptions. The symbol "-" means the solver failed (timeout or memout) on one or more instances.

The SmallComp dataset is the only one for which we obtain some results across the entire panel of solvers. Even with such dataset of small instances, as $\beta$ grows most solvers quickly stop responding within the alloted timeout. For example, even the best solver of SmallComp (i.e., Maximo) fails instances as $\beta>0.5$.

As expected, the larger the instances in the benchmark, the sooner complete solvers stop responding. More surprising is how

\footnotetext{
${ }^{5}$ Max-Sat competition web-site - http://maxsat.ia.udl.cat/introduction/
} 


\begin{tabular}{|c|c|c|c|c|c|c|c|c|c|c|}
\hline \multirow[b]{2}{*}{ Dataset } & \multirow[b]{2}{*}{$\# \mathrm{U}$} & \multirow[b]{2}{*}{$\# \mathrm{P}$} & \multirow[b]{2}{*}{ Density } & \multicolumn{2}{|c|}{ RBAC state } & \multirow[b]{2}{*}{ \#Excs } & \multicolumn{4}{|c|}{ Max-SAT encoding } \\
\hline & & & & $\left|R^{0}\right|$ & $\left|U A^{0}\right|+\left|P A^{0}\right|$ & & $\# V$ & $\# C$ & $\# C_{h}$ & $\# C_{s}$ \\
\hline SmallComp & 11 & 11 & 0.207 & 13 & 65 & 12 & $6.9 \cdot 10^{2}$ & $3 \cdot 10^{3}$ & $2.6 \cdot 10^{3}$ & $3.7 \cdot 10^{2}$ \\
\hline Domino & 79 & 231 & 0.039 & 71 & 1803 & 19 & $7.4 \cdot 10^{4}$ & $1.4 \cdot 10^{6}$ & $1.4 \cdot 10^{6}$ & $2.4 \cdot 10^{4}$ \\
\hline University & 493 & 56 & 0.143 & 71 & 8769 & 10 & $3.2 \cdot 10^{5}$ & $2.6 \cdot 10^{6}$ & $2.6 \cdot 10^{6}$ & $4.8 \cdot 10^{4}$ \\
\hline Firewall1 & 365 & 709 & 0.123 & 580 & 99713 & 32 & $1.9 \cdot 10^{7}$ & $1.9 \cdot 10^{8}$ & $1.9 \cdot 10^{8}$ & $7.2 \cdot 10^{5}$ \\
\hline
\end{tabular}

Table 5: Datasets in our benchmark. $\# U, \# P$, and Density are the number of users, permissions, and the percentage of assignments in the $U P A^{0}$ matrix, respectively, after the removal of $\#$ Excs exceptions. $\left|R^{0}\right|$ and $\left|U A^{0}\right|+\left|P A^{0}\right|$ are the number of roles and assignments in the initial RBAC state. \#V, \#C, \#C $C_{h}$, and $\# C_{s}$ are the number of variables, clauses, hard clauses, and soft clauses in the Max-SAT encoding, respectively.

quickly performance deteriorates: By the time we try to solve Firewall1, even the best solver doesn't return solutions unless $\beta=0$.

We conclude that it is not feasible to employ complete solvers to tackle real-world instances of our RBAC maintenance encoding, except perhaps for very small values of $\beta$.

Incomplete solvers. Let us shift our attention to state-of-the-art incomplete solvers, as represented by the best performers at MaxSAT 2016. We tested: Dsat, CCLS2015, CCEHC, OptiRiss, Dist, WPM3.

CCEHC [7] stands out here, because it is the only solver that: (1) showed a strong performance at Max-SAT 2016, (2) accepts command-line options to tune its solving behavior for industrial benchmarks, and (3) works as an anytime algorithm.

Let us first measure how CCEHC behaves in a case clearly beyond reach for complete solvers. We generate 90 maintenance instances of increasing size from Firewall 1 by selecting more and more of its users (i.e., rows); each instance is associated with a single exception to incorporate and generates a Max-SAT encoding of growing size. We ask CCEHC to incorporate the exception leaning strongly towards on optimized, simpler RBAC state $(\beta=0.8)$. Figure 2 shows the minimum timeout needed to obtain a feasible solution for these inputs as a function of their size.

While the minimum timeout grows exponentially, performances over instances in a real-world size range are acceptable; for example, it is possible to obtain a solution in less than one hour for a $337.2 \mathrm{MB}$

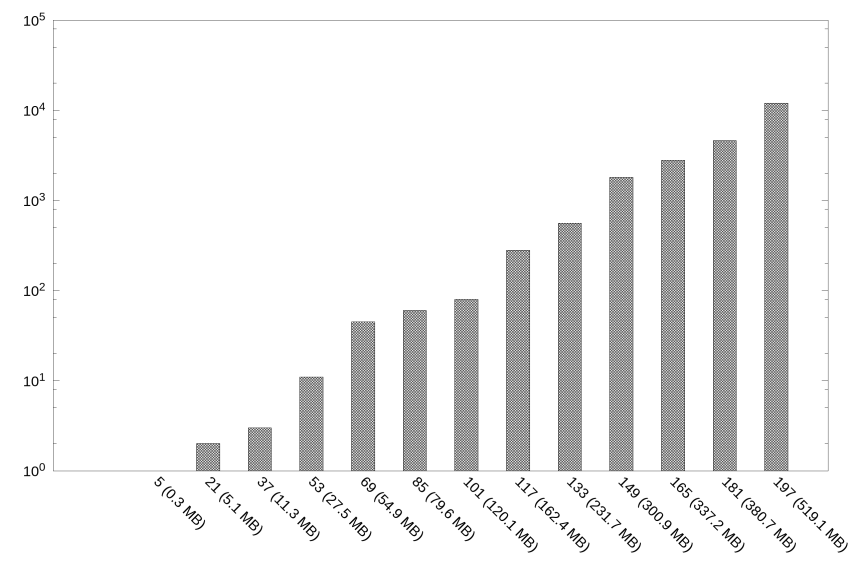

Figure 2: Minimum time to compute feasible solutions to Firewall1 ( $y$ axis, secs) as a function of the number of users ( $x$ axis). Along the $x$ axis we also note the size of the corresponding CNF encoding in Megabytes. formula that encodes the problem of incorporating one exception into an RBAC state with 165 users and 709 permissions.

But how good are these solutions? To provide an estimate we compare them to optimal solutions returned by complete solvers. As we have shown, Firewall1 is completely out of reach, so we resort to SmallComp. Given that hard constraints are always satisfied by feasible solutions from both complete and incomplete solvers, we focus on the ability of the incomplete solver to satisfy soft constraints. In particular, we compute the average weight of satisfied soft constraints over the total sum of input weights for the 12 different exceptions in the dataset. In Figure 3 we report this metric measured after CCEHC has worked for $t=2 \mathrm{sec}$ and then for $t=180 \mathrm{sec}$. We include the same metric computed on optimal solutions by complete solvers, which we could obtain for $\beta \leq 0.5$ (1 hour timeout). As expected, the complete solver outputs (slightly) better answers across the line, independently of $\beta$, but CCEHC is not far. These results, though quite comforting, have to be taken with a grain of salt because the small instances in SmallComp may not be representative of the general behavior of CCEHC.

4.2.3 Experimental Results. Incremental Max-SAT solvers seem capable of providing good approximate solutions within reasonable time on real-world instances. In the rest of this section we use them (namely, CCEHC) to explore the behavior of our encoding. We set $k^{-}=7$ and $k^{+}=2$ to prefer role reduction to matrix simplification.

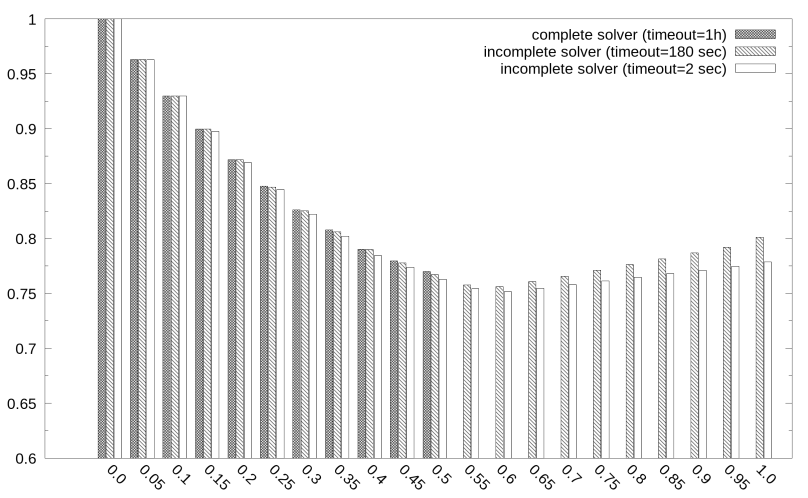

Figure 3: Average percentage of satisfied soft clauses (y axis) as a function of the balance $\beta$ (x axis) in the SmallComp dataset. 


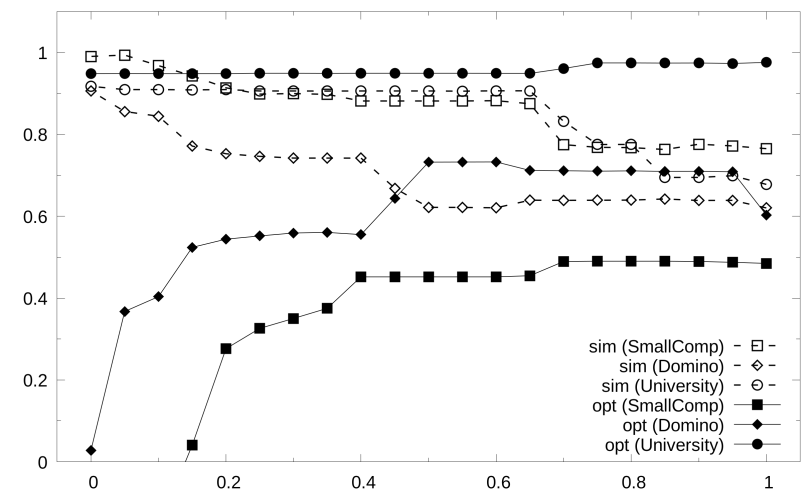

Figure 4: Average similarity and simplicity ( $y$ axis) as a function of the balance $\beta$ ( $x$ axis) for different datasets. Twenty-one values of $\beta \in[0,1]$ are sampled, at regular intervals (step 0.05).

Impact of $\beta$. The first thing we assess experimentally is the impact the value of $\beta$ has on the structure of the fixed RBAC state. Figure 4 shows that-for all datasets-similarity decreases (and simplicity increases) almost monotonically as $\beta$ grows. Figure 6 shows how the corresponding number of roles decreases on average by increasing the preference towards optimized solutions ( $\beta$ close to 1). Conversely, if the encoding leans towards preserving the original RBAC state ( $\beta$ close to 0 ) these numbers stay close to the input values. None of the instances in the largest of the four datasets could be solved within 1 hour: Firewall1 thus constitute a hard problem at present (see Conclusions). Overall, by tuning $\beta$, it seems actually possible to steer the quality of the solution towards similarity or simplicity during maintenance.

Impact of timeout. We show how the output RBAC state (simplicity, similarity) changes by granting more time to the solver, at different balance points. Figure 5 and 7 show the results for timeouts in $10 \mathrm{~s}-600 \mathrm{~s}$ on the dataset Domino (representative of the entire benchmark). Simplicity increases for $\beta \geq 0.25$ (we are optimizing the state as a side effect of the maintenance) while it drifts towards lower values when $\beta<0.25$ (the price we pay to avoid reworking the RBAC state too much as exceptions arrive). Conversely, similarity improves for low values of $\beta$ while it remains almost stable

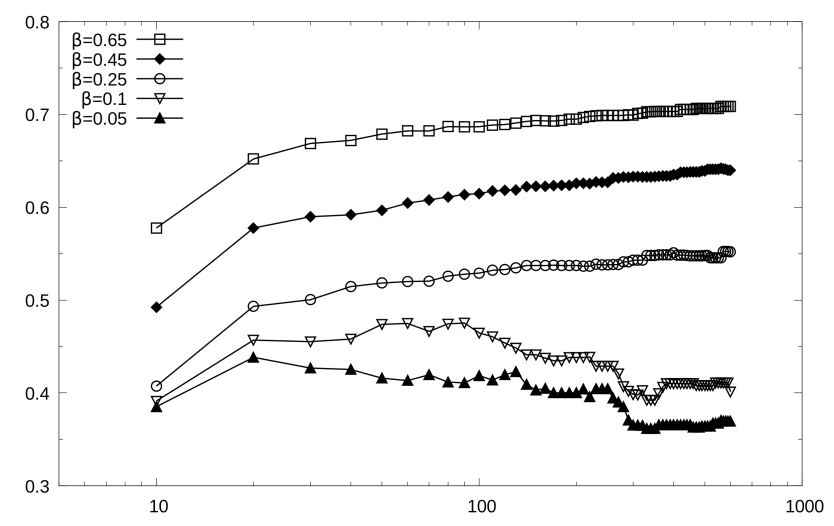

Figure 5: Average simplicity in Domino (y axis) as a function of the timeout ( $\mathrm{x}$ axis, secs) at difference balance points $(\beta)$.

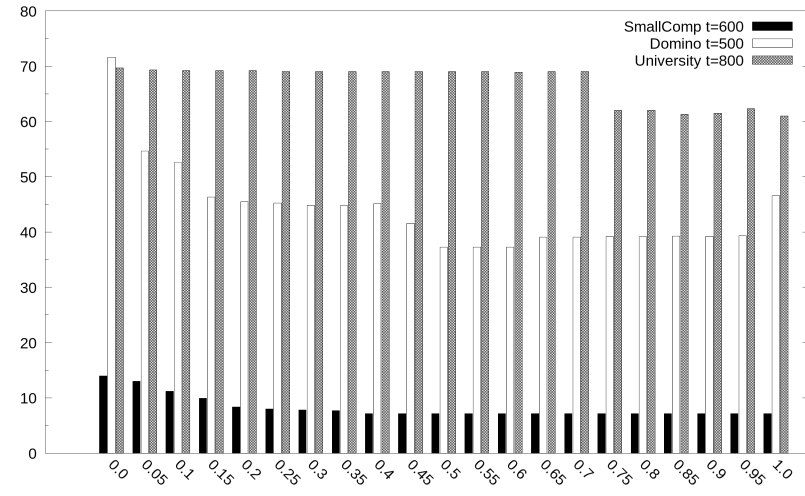

Figure 6: Average number of roles after incorporating exceptions (y axis) as a function of the balance $\beta$ ( $x$ axis) for different datasets.

for $\beta \geq 0.25$. It follows that if the interest towards simplification is high, it always pays to allot more reasoning time to the solver. Conversely, if the interest is in preserving the input state, increasing the reasoning time pays for $\beta<0.25$, where similarity increases at the expense of simplicity.

The order of exceptions. In these experiments, exceptions are incorporated sequentially, one-by-one, as they show up (though bulk incorporation is also possible, as already noted). We are interested in understanding how much the order in which they manifest affects the quality of the eventual state. In principle, the number of roles can either increase (small values of $\beta$ ) or decrease (large $\beta$ ). To confirm this, we select the Domino dataset and pick a string of 6 exceptions to be incorporated. We generate all their permutations (720) as incorporation sequences. For each sequence, we record the final number of roles, assignments, simplicity and similarity to the initial state (that has 73 roles). Figure 8 shows the distribution generated by 715 paths (each solvable in 60 seconds) with four different $\beta$ configurations. While by construction the final states are equivalent in terms of Permission-to-User assignments, the average value and the variance of the distributions are profoundly impacted by $\beta$; the variance in particular widens as $\beta$ goes close to 0 ; for example, with $\beta=0.1$ the number of roles ranges from 28 to

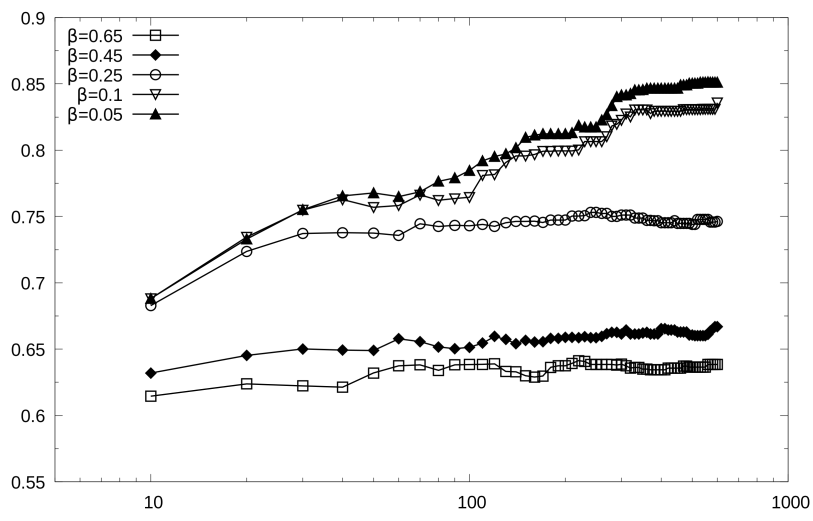

Figure 7: Average similarity in Domino (y axis) as a function of the timeout ( $x$ axis, secs) at difference balance points $(\beta)$. 


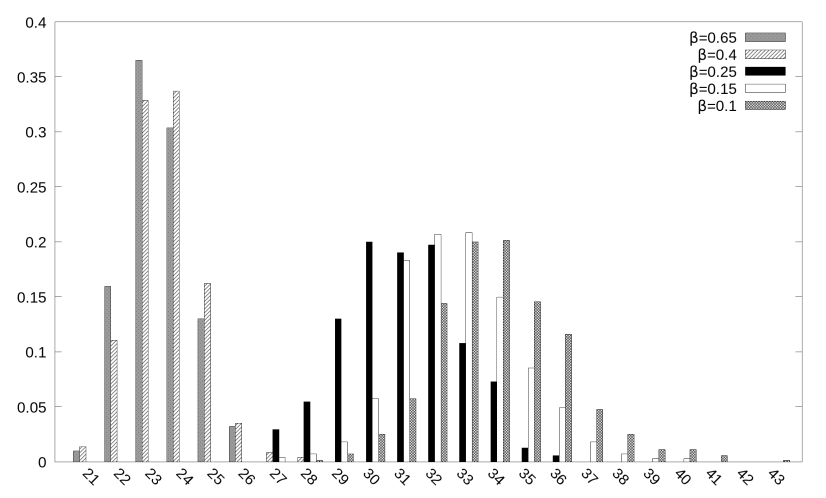

Figure 8: Distribution of the number of roles in Domino (x axis) after incorporating 6 exceptions in all possible orderings.

43 , corresponding respectively to a $61.6 \%$ to $41.1 \%$ reduction with respect to initial RBAC state.

This behavior suggests that-independently of the order in which exceptions arrive-by leveraging $\beta$ the RBAC administrator can direct the state over time to become simpler, to accumulate "clutter," or to remain stable in terms of number of roles.

\section{RELATED WORK}

The literature on Role Mining and RBAC state tuning is quite extensive. The works most closely related to this paper are [5, 13, 16, 17]. These approaches are similar to ours in that they aim at reconfiguring an existing set of roles with the goal of simplifying them.

In [16] the simplification procedure works as follows: A candidate set of new roles are iteratively generated starting from the trivial role set (one role per permission) and combining them in several ways (pairwise, etc.). The candidate set is kept consistent with the input RBAC state, i.e., each old role must be representable as the union of some new roles. Individual candidates with the lowest "management cost" are preferred, where the management cost is the sum of a fixed component (the same for all roles) plus a variable component which depends on the role cardinality and granularity. A parameter is introduced to balance their relative importance.

The approach presented in [13] more directly balances the quality of the new RBAC state against its similarity to the current one. It first applies a canonical role mining algorithm (Fast Miner) to generate a starting set of candidates. Then it iteratively evaluates roles according to a fitness function and selects the best ones until a solution is obtained that covers all the Permission-to-User assignments of the input RBAC state. The fitness function is a weighted sum of a measure of similarity to the original roles and a measure of coverage of the input Permission-to-User matrix. This algorithm has been extended to work with role hierarchies $[4,11]$.

A different perspective is adopted in [17]. This procedure analyzes logs of actual permission utilizations over time and exploits this data to inform the next RBAC state. There are two phases: The first phase applies a variant of the subset enumeration technique of Fast Miner to iteratively generate a set of roles, which are then sorted according to a fitness function. The fitness function is where the traces of real permission usage come into play: It aims at balancing similarity to the original roles with "homogeneity" to actual permission utilization. At each iteration, top ranked roles which cover the input Permission-to-User matrix are selected; this is repeated until fixpoint (two subsequent steps result in the same set of roles) or until a maximum number of iterations is reached. The second phase then assigns roles to users based on a heuristic process which aims to contain redundancy.

A first difference between our approach and $[13,16]$ is that those procedures ignore how the simplified Permission-to-Role matrix they output will impact (in terms of administration costs) the correspondingly adjusted Permission-to-User assignments. This is evident because at no point those procedures take into account the UA matrix or how complex it would be for the administrator to edit it. Furthermore, in [16] the management cost of a set of roles is defined by only looking at roles per se, and not at the distance from the original. Overall, the link between the input matrices and the output matrices is very indirect: Both couples represent the same Permission-to-User relations, but the difficulty for an administrator to transform $U A^{0}$ into $U A$ and $P A^{0}$ into $P A$ is not modeled. Conversely, our encoding explicitly captures the difference of $U A$ from $U A^{0}$ and $P A$ from $P A^{0}$, so it relates more directly to the amount of work an administrator will have to do.

Another major difference from $[13,16,17]$ is that our approach captures the RBAC optimization problem declaratively within a logic formalism, and neatly separates the declaration of the constraints and objective function from the search for an optimal solution. This provides several benefits and additional guarantees versus employing custom multi-phase heuristics. One advantage is that future breakthroughs from the community developing complete (and incomplete) Max-SAT solvers will result in an improved RBAC maintenance process without any change to our encoding. Another advantage is that the mechanism controlling the trade-off between quality and similarity provides strong guarantees about the output RBAC state; for example, for $\beta=0$ we are sure that the output RBAC state is the least variation to the input that is capable of accommodating the intervening exception, whereas for $\beta=1$ we have the guarantee that the output state is optimal w.r.t. a certain metric and that the input state has been completely ignored ${ }^{6}$.

A logic-based formalization of the RBAC state similar to ours is presented in [5]. In that case, the Satisfiability Modulo Theory (SMT) framework is adopted; the theory used to expand the expressive power of SAT is Integer Linear Programming (ILP), employed to capture certain boolean-unfriendly quality metrics. The paper shows how to exploit SMT to solve several role mining variants, including one that generates an RBAC state optimal in terms of a combination of WSC [9] and similarity to an input RBAC state. To this end, a heuristic technique is presented to iteratively generate role sets, which are evaluated according to a fitness function that balances the conflicting WSC-vs-similarity objectives. The complete RBAC state is thus taken into account, and configurations that disrupt the Role-to-User assignments are penalized. Other

${ }^{6}$ It is worth noticing that while the encoding we propose offers these guarantees, we may lose them by solving the resulting problems with incomplete solvers. 
variants consider the permission usage or the RBAC hierarchy as optimization metrics, resulting in a hybrid role mining approach.

The major difference between this approach and ours (beyond the definitions of certain quality metrics) is that we embrace the inherent nature of the role maintenance process as an optimization procedure by using a logic framework (Max-SAT) meant to optimize an objective function, whereas Satisfiability Modulo Theory (SMT) is a decision procedure meant to prove the consistency of statements. As a result, while the entire problem is captured by a single Max-SAT instance and the reasoning/searching/optimization stage is decoupled and offloaded to an external solver, [5] generates a long sequence of SMT problems in the context of a complex, custom algorithm to achieve the trade-off between quality and similarity. Another difference is that instead of generating candidate role sets and testing them against the original RBAC state to assess their similarity, we directly embed the original RBAC state as-is in the encoding together with penalties for diverging from it.

Another key difference from previous approaches is in when and how the RBAC reconfiguration is supposed to happen. Previous algorithms are presented as off-line procedures; administrators are supposed to first let the complexity of the RBAC state increase, perhaps by creating several ad-hoc new roles to quickly fix exceptions. Then, from time to time, a procedure is applied to simplify everything. Large discontinuities are acceptable at these "reconfiguration points". Conversely, the approach presented here can be leveraged to continuously introduce small optimizing changes: Applied on-line, in an exception-driven way, it steers the trajectory of the RBAC state towards simplicity without any costly or stakeholder-adverse update.

\section{CONCLUSION AND FUTURE WORK}

We presented a novel approach to perform maintenance of RBAC states, that is, to incorporate exceptions as they show up and improve the quality of the role model along the way. Based upon generating Max-SAT instances and solving them via state-of-theart solvers, this method seems capable of dealing with real-life instances.

We are motivated to pursue optimized RBAC maintenance by actual issues at our company. Seldom there is the time and opportunity to perform large, impactful RBAC updates; conversely, errors show up on a daily basis, and if any push towards optimization can be exerted during maintenance, the RBAC state may actually start to converge to an (almost) optimal version of itself.

Directions for future work are as follows. Large datasets (e.g., Firewall1) still require too much time to solve; improvements to the encodings and/or the solving stage are of the essence here ${ }^{7}$. We haven't fully investigated yet how our technique behaves in the case of permissions in excess, to be withdrawn; these cases are indeed more difficult to capture automatically and we have less examples at our disposal. An optimal, fully-specified (and automatically synthesized) course of action for the RBAC administrator to implement a fix into an RBAC management system in use is another objective of interest, which seems within reach.

\footnotetext{
${ }^{7}$ We submitted our datasets to the organizers of the Max-SAT competition to provide them with challenging real-world instances on which to compare solvers.
}

\section{ACKNOWLEDGMENTS}

The authors thank Dr. Chuan Luo for supplying us with an improved and configurable version of the solver $C C E H C$; Dr. Carlos Ansótegui for the solvers Dsat and WPM3; Dr. Shaowei Cai for Dist.

Several colleagues from the Bank of Italy offered help: A special thanks goes to Domenico Pansini (security administrator) for providing us with examples and insights on role maintenance in a real-world setting; Oliver Giudice, Giuseppe Galano, and Roberto Favaroni (ART team) offered a great deal of support in setting up and configuring the computational infrastructure we used for our experiments; Arturo Baldo was the first to suggest the opportunity to investigate formal approaches to role maintenance. Finally, the anonymous referees helped us to produce a better and more readable contribution.

\section{REFERENCES}

[1] Alessandro Colantonio, Roberto Di Pietro, and Alberto Ocello. 2008. A CostDriven Approach to Role Engineering. In Proceedings of the 2008 ACM symposium on Applied computing. ACM, 2129-2136.

[2] Stephen A. Cook. 1971. The Complexity of Theorem-Proving Procedures. In Proceedings of the third annual ACM symposium on Theory of computing. ACM, 151-158.

[3] David F. Ferraiolo, Ravi Sandhu, Serban Gavrila, D. Richard Kuhn, and Ramaswamy Chandramouli. 2001. Proposed NIST Standard for Role-Based Access Control. ACM Transactions on Information and System Security (TISSEC) 4, 3 (2001), 224-274.

[4] Qi Guo, Jaideep Vaidya, and Vijayalakshmi Atluri. 2008. The Role Hierarchy Mining Problem: Discovery of Optimal Role Hierarchies. In Computer Security Applications Conference, 2008. ACSAC 2008. Annual. IEEE, 237-246.

[5] Jafar Haadi Jafarian, Hassan Takabi, Hakim Touati, Ehsan Hesamifard, and Mohamed Shehab. 2015. Towards a General Framework for Optimal Role Mining: A Constraint Satisfaction Approach. In Proceedings of the 20th ACM Symposium on Access Control Models and Technologies. ACM, 211-220.

[6] David S. Johnson. 1973. Approximation Algorithms for Combinatorial Problems. In Proceedings of the fifth annual ACM symposium on Theory of computing. ACM, $38-49$.

[7] Chuan Luo, Shaowei Cai, Kaile Su, and Wenxuan Huang. 2017. CCEHC: An Efficient Local Search Algorithm for Weighted Partial Maximum Satisfiability. Artificial Intelligence 243 (2017), 26-44.

[8] Barsha Mitra, Shamik Sural, Jaideep Vaidya, and Vijayalakshmi Atluri. 2016. A Survey of Role Mining. ACM Computing Surveys (CSUR) 48, 4 (2016), 50

[9] Ian Molloy, Hong Chen, Tiancheng Li, Qihua Wang, Ninghui Li, Elisa Bertino, Seraphin Calo, and Jorge Lobo. 2008. Mining Roles with Semantic Meanings. In Proceedings of the 13th ACM symposium on Access control models and technologies. ACM, 21-30.

[10] Marco Mori and Marco Benedetti. 2018. Web page with downloadable experiments and datasets. (2018). Retrieved April 19, 2018 from https://bancaditalia. github.io/sacmat2018/

[11] Hassan Takabi and James B.D. Joshi. 2010. StateMiner: An Efficient SimilarityBased Approach for Optimal Mining of Role Hierarchy. In Proceedings of the 15th ACM symposium on Access control models and technologies. ACM, 55-64.

[12] Grigori S. Tseitin. 1983. On the complexity of derivation in propositional calculus. In Automation of reasoning. Springer, 466-483.

[13] Jaideep Vaidya, Vijayalakshmi Atluri, Qi Guo, and Nabil Adam. 2008. Migrating to Optimal RBAC with Minimal Perturbation. In Proceedings of the 13th ACM symposium on Access control models and technologies. ACM, 11-20.

[14] Heidi L. Wachs. 2014. How to Succeed With Role Management and Avoid Common Pitfalls. Technical Report ID: G00262708. Gartner Technical Professional Advice.

[15] Heidi L. Wachs. 2015. Take Control of Enterprise Role Management. Technical Report ID: G00262285. Gartner Article.

[16] Hao Xia, Milind Dawande, and Vijay Mookerjee. 2014. Role Refinement in Access Control: Model and Analysis. INFORMS fournal on Computing 26, 4 (2014), 866-884.

[17] Wen Zhang, You Chen, Carl Gunter, David Liebovitz, and Bradley Malin. 2013. Evolving Role Definitions Through Permission Invocation Patterns. In Proceedings of the 18th ACM symposium on Access control models and technologies. ACM, 3748. 